Silva, RM, Rocha, JF, Medeiros, LADM, Maia, LS, Goes, VN, Oliveira, OL, Fragoso, LNM, Moura, ABR, Penha, ES, Guênes, GMT, Almeida, MSC \&Figueiredo, CHMC (2020). Dental students knowledge of dental autotransplantation. Research, Society and Development, 9(7): 1-16, e641974632.

\title{
Conhecimento de estudantes de Odontologia sobre autotransplante dentário
}

Dental students knowledge of Dental autotransplantation

\section{Conocimiento de estudiantes de Odontología sobre el auto-trasplante dental}

Recebido: 18/05/2020 | Revisado: 20/05/2020 | Aceito: 22/05/2020 | Publicado: 30/05/2020

Regina Mendes da Silva

ORCID: https://orcid.org/0000-0002-8032-3998

Universidade Federal de Campina Grande, Brasil

E-mail: reginamendes16@hotmail.com

Julierme Ferreira Rocha

ORCID: https://orcid.org/0000-0001-9025-5661

Universidade Federal de Campina Grande, Brasil

E-mail: juliermerocha@ hotmail.com

Luanna Abílio Diniz Melquíades de Medeiros

ORCID: https://orcid.org/0000-0002-1630-3968

Universidade Federal de Campina Grande, Brasil

E-mail: luannaabiliod@gmail.com

Laís Sousa Maia

ORCID: https://orcid.org/0000-0003-1742-8989

Universidade Federal de Campina Grande, Brasil

E-mail: laissousahmaia@gmail.com

Vitor Nascimento Goes

ORCID: https://orcid.org/0000-0001-5163-1126

Universidade Federal de Campina Grande, Brasil

E-mail: vitor9511goes@ hotmail.com

Ocimar Lopes de Oliveira

ORCID: https://orcid.org/0000-0002-0573-4578

Universidade Federal de Campina Grande, Brasil

E-mail: ocimarlp99@gmail.com 
Lukas Natã Mendes Fragoso

ORCID: https://orcid.org/0000-0001-7003-7173

Universidade Federal de Campina Grande, Brasil

E-mail: fragoso.luks@gmail.com

Ana Beatriz Rodrigues Moura

ORCID: https://orcid.org/0000-0003-0006-148X

Universidade Federal de Campina Grande, Brasil

E-mail: ana_beatriz_882@hotmail.com

Elizandra Silva da Penha

ORCID: https://orcid.org/0000-0001-6264-5232

Universidade Federal de Campina Grande, Brasil

E-mail: elizandrapenha@ hotmail.com

Gymenna Maria Tenório Guênes

ORCID: https://orcid.org/0000-0002-5447-0193

Universidade Federal de Campina Grande, Brasil

E-mail: gymennat@yahoo.com.br

Manuella Santos Carneiro Almeida

ORCID: https://orcid.org/0000-0002-5849-6972

Universidade Federal de Campina Grande, Brasil

E-mail: manuellacarneiro@ hotmail.com

Camila Helena Machado da Costa Figueiredo

ORCID: https://orcid.org/0000-0002-1340-4042

Universidade Federal de Campina Grande, Brasil

E-mail: camila_helena_@hotmail.com

\section{Resumo}

Objetivo: Identificar o nível de conhecimento dos alunos do Curso de Odontologia da Universidade Federal de Campina Grande, campus de Patos-Paraíba, a respeito do autotransplante dentário. Métodos: Este estudo foi do tipo transversal, observacional, com abordagem indutiva, descritivo, adotando como estratégia de coleta de dados o questionário específico, aplicados com 143 graduandos regularmente matriculados do $5^{\circ}$ ao $10^{\circ}$ período do curso de odontologia, os quais já haviam cursado a disciplina teórica de cirurgia, entre o ano de 2019 e 2020. Aspectos abordados: caracterização dos estudantes e informações relacionadas ao autotransplante dentário (conceito, indicações, contraindicações e fatores que 
influenciam no sucesso do tratamento). Resultados: A maioria dos entrevistados foi do gênero feminino. Observou-se um nível satisfatório de conhecimento sobre as indicações, contra-indicações, vantagens e necessidade de ausência de carga do dente autotransplantado.

Conclusão: Apesar de não ser um procedimento comum na prática clínica e do ensino odontológico, dentro da metodologia empregada nesse estudo, foi possível observar um nível de conhecimento satisfatório dos estudantes de odontologia da UFCG, Patos-Paraíba acerca do autotransplante dentário.

Palavras-chave: Odontologia; Reabilitação bucal; Transplante autólogo.

\begin{abstract}
Objective: To identify the level of knowledge of students of the Dentistry Course at the Federal University of Campina Grande, campus of Patos, regarding dental autotransplantation as a treatment for oral rehabilitation. Methods: This was a cross-sectional, observational study, with an inductive, descriptive approach, adopting a specific questionnaire as a strategy for data collection, applied to 143 graduates regularly enrolled from the 5 th to the 10 th period of the dentistry course, between the year 2019 and 2020. Aspects covered: characterization of students and information related to dental autotransplantation (concept, indications, contraindications and factors that influence the success of treatment). Results: Most of the interviewees were female. There was a satisfactory level of knowledge about the indications, contraindications, advantages and the need for no loading of the self-transplanted tooth. Conclusion: Despite not being a common procedure in clinical practice and dental education, within the methodology used in this study, it was possible to observe a satisfactory level of knowledge among dentistry students at UFCG, Patos-Paraíba about dental autotransplantation.
\end{abstract}

Keywords: Dentistry, Mouth rehabilitation, Autoulogous transplantation.

\title{
Resumen
}

Objetivo: Identificar el nivel de conocimiento de los estudiantes del Curso de Odontología en la Universidad Federal de Campina Grande, campus de Patos, sobre el autotrasplante dental como tratamiento para la rehabilitación oral. Métodos: Este fue un estudio transversal, observacional, con un enfoque inductivo y descriptivo, que adoptó un cuestionario específico como estrategia de recolección de datos, aplicado a 143 estudiantes de pregrado matriculados regularmente del $5^{\circ}$ al $10^{\circ}$ período del curso de odontología, que ya habían asistido al curso. disciplina teórica de la cirugía, entre 2019 y 2020. Aspectos cubiertos: caracterización de 
estudiantes e información relacionada con el autotrasplante dental (concepto, indicaciones, contraindicaciones y factores que influyen en el éxito del tratamiento). Resultados: La mayoría de los entrevistados eran mujeres. Hubo un nivel de conocimiento satisfactorio sobre las indicaciones, contraindicaciones, ventajas y la necesidad de no cargar el diente autotrasplantado. Conclusión: a pesar de no ser un procedimiento común en la práctica clínica y la educación dental, dentro de la metodología utilizada en este estudio, fue posible observar un nivel satisfactorio de conocimiento entre los estudiantes de odontología en UFCG, Patos-Paraíba sobre el autotrasplante dental.

Palabras clave: Odontología; Rehabilitación bucal; Trasplante autólogo.

\section{Introdução}

Muitos são os fatores que levam a perda dentária, sendo a cárie considerada o fator predominante, todavia problemas periodontais e episódios de trauma também podem ocorrer. Quando a perda do dente acontece em indivíduos jovens e em desenvolvimento é necessária uma adequada substituição dentária ou meios que, preservem o espaço no arco para uma provável e futura reabilitação, devolvendo assim estética e funcionalidade ao paciente (Andreasen,Schwartz, Kofoed \& Daugaard-Jensen, 2009; Gilijamse, Baart, Wolff, Sándor \& Forouzanfar, 2016).

A reabilitação oral representa um grande desafio à odontologia, principalmente em perdas dentárias precoces, pelos fatores estéticos e funcionais associados. Comumente, esta reabilitação vem sendo realizada por meio de próteses removíveis, fixas ou implantossuportadas. Todavia, essas alternativas terapêuticas resultam usualmente em desconforto durante o assentamento da prótese e no ato mastigatório, desgastes de dentes saudáveis ou estão contraindicadas em crianças e adolescentes até 17 anos em fase de crescimento ósseo (Duarte et al., 2017; Nagori, Bhutia, Roychoudhury \& Pandey, 2014).

O autotransplante dentário é um procedimento odontológico que substitui um dente perdido, como alternativa a reposições protéticas. Estudos vêm sendo realizados, confirmando que o transplante dental autógeno é um procedimento seguro, rápido e economicamente viável e que, a depender de uma associação de uma boa técnica cirúrgica, atenção e habilidade do cirurgião e da cooperação do paciente, o prognóstico é favorável (Mikami et al., 2014).

Em razão disso, o propósito do presente estudo é identificar o nível de conhecimento dos alunos do Curso de Odontologia da Universidade Federal de Campina Grande (UFCG), campus de Patos, a respeito do autotransplante dentário como tratamento para reabilitação 
oral.

\section{Metodologia}

Este estudo foi do tipo transversal, observacional, com abordagem indutiva e descritiva, adotando como estratégia de coleta de dados o questionário específico.

$\mathrm{O}$ universo foi composto pelos alunos regularmente matriculados do $5^{\circ}$ ao $10^{\circ}$ período do Curso de Odontologia da Universidade Federal de Campina Grande, campus de Patos, no período compreendido entre o ano de 2019 e 2020.

O cálculo amostral considerou um grau de confiança de $95 \%$, poder de teste de $50 \%$ e erro aceitável de 5\%, em um universo de 350 estudantes, obteve-se uma amostra de 143 participantes.

Para a participação dos graduandos nessa pesquisa foram considerados como critérios de inclusão: Ser estudante de odontologia da Universidade Federal de Campina Grande, campus de Patos-Paraíba, está regularmente matriculado entre o $5^{\circ}$ e o $10^{\circ}$ período e ter cursado a disciplina de cirurgia; está presente na sala de aula no dia da coleta; e aceitar participar da pesquisa mediante a assinatura do Termo de Consentimento Livre e Esclarecido (TCLE).

A coleta de dados foi realizada por um pesquisador, por meio de um questionário estruturado direcionado aos graduandos do curso de Odontologia da Universidade Federal de Campina Grande, campus de Patos, englobando questões sobre autotransplante dentário.

Os seguintes aspectos foram abordados: caracterização dos estudantes (idade, sexo, período do curso) e informações relacionadas ao autotransplante dentário (conceito da técnica, indicações, contraindicações e fatores que influenciam no sucesso do tratamento).

Previamente, foi realizado um estudo piloto com 5 questionários, visando testar os instrumentos da pesquisa para possíveis adequações.

Os questionários com respostas incompletas ou rasuradas foram excluídos da pesquisa.

Após coletados, os dados foram registrados na forma de banco de dados do programa de informática SPSS (Statistical Package for Social Sciences) para Windows, versão 13.0, e foram trabalhados pela estatística descritiva.

O projeto de pesquisa foi enviado para o Comitê de Ética em Pesquisa com Seres Humanos da Faculdade Integrada de Patos (FIP) e aprovado sob o número 3.327.701. 


\section{Resultados}

Foram avaliados 143 questionários com graduandos do Curso de Odontologia da Universidade Federal de Campina Grande, campus de Patos, no período de 2019 a 2020 . A maioria dos participantes é do gênero feminino (96 participantes).

A Tabela 1 aponta os resultados acerca da principal indicação de faixa etária e leito receptor mais prevalente do transplante dentário autógeno.

Tabela 1: Distribuição da amostra quanto ao conhecimento acerca da principal indicação e leito receptor mais prevalente do transplante dentário autógeno. Patos/PB, 2019.

O transplante dentário é principalmente indicado para:

\section{Crianças e adolescentes}

Idosos

n

19
$\%$

13,3
Não sei

n

41

A maior prevalência na realização de transplantes autógenos ocorre do terceiro molar para o lugar do primeiro ou segundo molar?

Sim

$\mathbf{n}$

85
$\%$

59,4
Não

n

5
$\%$

3,5
Não sei
$\%$

53
$\%$

28,7

Fonte: Autores.

A Tabela 2 aponta os resultados sobre o grau de conhecimento acerca das técnicas cirúrgicas que podem ser utilizadas no transplante dentário autógeno. 
Tabela 2: Distribuição da amostra quanto ao conhecimento acerca das técnicas operatórias do transplante dentário autógeno. Patos/PB, 2019.

O transplante dentário pode ser realizado pela técnica convencional ou imediata ou pela técnica mediata ou tardia?

Sim

\section{n}

39

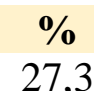

27,3
Não

n

Não sei

$\begin{array}{ccc}\% & \mathbf{n} & \% \\ 7 & 94 & 65,7\end{array}$

Fonte: Autores.

O conhecimento dos graduandos acerca das contraindicações e fatores que podem exercer influência sobre o transplante dentário autógeno está evidenciadas na Tabela 3.

Tabela 3: Distribuição da amostra quanto ao conhecimento acerca das contraindicações e fatores que podem exercer influência no transplante dentário autógeno. Patos/PB, 2019.

A periodontite agressiva é uma contraindicação para a realização do transplante dentário?

Sim

n

120

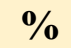

83,9

Não

Não sei

Quantidade de osso insuficiente no leito receptor é uma contraindicação para a realização do transplante dentário?

Sim

Não

Não sei

$\begin{array}{cccccc}\mathbf{n} & \boldsymbol{\%} & \mathbf{n} & \boldsymbol{\%} & \mathbf{n} & \boldsymbol{\%} \\ 124 & 86,7 & 4 & 2,8 & 15 & 10,5\end{array}$

Higiene oral deficiente é uma contraindicação para a realização do transplante dentário?

Sim

Não

Não sei

$\begin{array}{cccccc}\mathbf{n} & \boldsymbol{\%} & \mathbf{n} & \boldsymbol{\%} & \mathbf{n} & \boldsymbol{\%} \\ 113 & 79 & 5 & 3,5 & 25 & 17,5\end{array}$

A idade do paciente pode influenciar no sucesso do transplante dentário? Sim

Não

Não sei

$\begin{array}{cccccc}\mathbf{n} & \mathbf{\%} & \mathbf{n} & \mathbf{\%} & \mathbf{n} & \mathbf{\%} \\ 118 & 82,5 & 0 & 0 & 25 & 17,5\end{array}$

A anatomia e condição do dente doador podem influenciar no sucesso do transplante dentário?

Sim

n

129

$\%$
90,2

n

1

Não

Não sei

Fonte: Autores. 
Quando questionados se já haviam assistido ou mesmo realizado a uma cirurgia de transplante dentário autógeno, apenas 5 graduandos $(3,5 \%)$ responderam que sim.

Ao serem questionados se o transplante dentário autógeno apresenta benefícios ao paciente, 132 graduandos $(92,3 \%)$ responderam que sim, $1(0,7 \%)$ respondeu que não e 10 (7\%) não souberam responder a essa questão.

A Tabela 4 aponta os resultados acerca dos benefícios que o transplante dentário autógeno pode proporcionar.

Tabela 4: Distribuição da amostra quanto ao conhecimento acerca dos benefícios proporcionados pelo transplante dentário autógeno. Patos/PB, 2019.

\begin{tabular}{|c|c|c|c|c|c|c|c|c|c|c|c|}
\hline \multicolumn{12}{|c|}{ Aponte quais benefícios o transplante dentário apresenta. } \\
\hline \multicolumn{2}{|c|}{$\begin{array}{c}\text { Reabilitação de } \\
\text { perdas } \\
\text { dentárias }\end{array}$} & \multicolumn{2}{|c|}{$\begin{array}{l}\text { Técnica } \\
\text { cirúrgica } \\
\text { simples }\end{array}$} & \multicolumn{2}{|c|}{$\begin{array}{l}\text { Independe } \\
\text { de trabalho } \\
\text { protético }\end{array}$} & \multicolumn{2}{|c|}{$\begin{array}{l}\text { Não necessita de } \\
\text { complexos } \\
\text { equipamentos } \\
\text { odontológicos }\end{array}$} & \multicolumn{2}{|c|}{$\begin{array}{c}\text { Baixo } \\
\text { custo }\end{array}$} & \multicolumn{2}{|c|}{$\begin{array}{c}\text { Não } \\
\text { sei }\end{array}$} \\
\hline $\mathbf{n}$ & $\%$ & $\mathbf{n}$ & $\%$ & $\mathbf{n}$ & $\%$ & $\mathbf{n}$ & $\%$ & $\mathbf{n}$ & $\%$ & $\mathbf{n}$ & $\%$ \\
\hline 127 & 88,8 & 28 & 19,6 & 54 & 37,8 & 39 & 27,3 & 48 & 33,6 & 10 & 7 \\
\hline
\end{tabular}

Fonte: Autores.

O conhecimento dos graduandos acerca dos fatores que podem influenciar o sucesso do transplante dentário autógeno está apresentado na Tabela 5. 
Tabela 5: Distribuição da amostra quando ao conhecimento acerca dos fatores que influenciam o sucesso do transplante dentário autógeno. Patos/PB, 2019.

\section{É necessário que se realize tratamento endodôntico em todo dente transplantado?}

Sim

$\mathbf{n}$

21
Não

$\begin{array}{ccc}\boldsymbol{\%} & \mathbf{n} & \mathbf{\%} \\ 21 & 92 & 64,3\end{array}$

A esplintagem do dente transplantado é obrigatória?

Sim

$\%$

14,7

Não

Não sei

$\begin{array}{cccccc}\mathbf{n} & \boldsymbol{\%} & \mathbf{n} & \mathbf{\%} & \mathbf{n} & \boldsymbol{\%} \\ 31 & 21,7 & 10 & 7 & 102 & 71,3\end{array}$

É necessário que o dente submetido ao transplante fique livre de cargas mastigatórias no período inicial?

Sim

$\mathbf{n}$

77

(2)

\section{$\%$}

53,8

Não

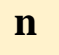

9
$\%$

6,3
Não sei

57
$\%$

39,9

Fonte: Autores.

\section{Discussão}

O autotransplante dentário é uma manobra cirúrgica em que o dente a ser transplantado é submetido a uma avulsão do seu local de origem e implantado em outro alvéolo natural ou preparado cirurgicamente (Queiroz et al., 2016).

Esta técnica é principalmente indicada para crianças e adolescentes que precisam substituir dentes perdidos, seja decorrente de ausência congênita, ou seja, por: perdas prematuras, traumatismos, iatrogenias ou, quando o tratamento restaurador protético estiver inviabilizado por motivos socioeconômicos (Correio et al., 2015).

A literatura reporta que o autotransplante tem prognóstico mais favorável quando realizado em pacientes jovens, sem problemas de saúde sistêmicos graves, que concordem 
com a intervenção e sejam cooperantes. Estudos indicam que em pacientes de 3 a 14 anos a vitalidade pulpar é preservada em 90 a 96\% casos (Donado, 2007).

$\mathrm{Na}$ presente pesquisa, ao serem questionados sobre a faixa etária que apresenta principal indicação no autotransplante dentário, 28,7\% dos graduandos afirmou não saber a resposta correta. Contudo, alguns autores relatam que se alcançam resultados semelhantes em qualquer idade, seja em dentes colocados em alvéolos naturais, seja em alvéolos artificiais criados pelo cirurgião (Ibid, 2007).

A maior prevalência na realização de autotransplantes ocorre do terceiro molar para o lugar do primeiro ou segundo molar. A principal causa do sucesso dessa técnica consiste no desenvolvimento tardio dos terceiros molares em relação aos demais dentes (Queiroz et al., 2016). Ao serem questionados sobre o leito receptor mais prevalente, 59,4\% dos graduandos de odontologia da UFCG responderam que estava correta a afirmação de que a maior realização de transplantes autógenos ocorre do terceiro molar para o lugar do primeiro ou segundo molar. No estudo de Kokai et al. (2015), o qual avaliou retrospectivamente 100 dentes autotransplantados em 89 pacientes, há uma prevalência de pré-molares como dentes doadores $(54 \%)$.

O autotransplante dentário pode ser realizado pela técnica convencional ou imediata em uma única etapa, que consiste em realizar a extração do dente a ser transplantado e o preparo da cavidade óssea alveolar para o qual esse dente será transferido. A outra opção é pela técnica mediata ou tardia, em duas etapas, na qual o alvéolo cirúrgico é preparado na primeira etapa. Após um período inicial de cicatrização de aproximadamente 14 dias, realizase, na segunda etapa, a exodontia e o transplante. A técnica mediata também pode ser utilizada nas situações de perdas dentais precoces ou congênitas, onde, obrigatoriamente, há necessidade de criar um alvéolo cirurgicamente para receber o dente autotransplantado (Peixoto, Melo \& Santos, 2013; Yu, Jia, Lv \& Qiu, 2017). A maioria dos estudantes respondeu não conhecer essas duas técnicas.

A área receptora do dente transplantado necessita de alguns critérios, sendo considerada ideal aquela com altura e largura suficientes para acolher o dente doador em sua totalidade, o que favorece a cicatrização caso esteja presente ainda tecido do ligamento periodontal no alvéolo após extração do dente perdido. Todavia, é possível melhorar o local receptor aumentando o alvéolo de maneira cirúrgica, e ocasionalmente poderá ser necessário, em dentes da arcada superior, um levantamento do seio maxilar. Havendo quantidade óssea vestíbulo-lingual insuficiente, poderá haver reabsorção do rebordo alveolar na área receptora. (Aslan, Uçuncu \& Dogan, 2010). 
As principais contraindicações do autotransplante dentário são as mesmas de qualquer intervenção cirúrgica reabilitadora: quantidade óssea insuficiente no leito receptor, periodontite agressiva e higiene oral deficiente. O sucesso da técnica pode também ser influenciado pela idade do paciente, desenvolvimento, anatomia e condições do dente doador e pela técnica cirúrgica empregada (Correio et al., 2015).

Acerca de quantidade de osso insuficiente, $86,7 \%$ dos questionados apontaram-na como uma contraindicação do autotransplante dentário. O suporte ósseo é fundamental para favorecer a estabilização dentária e o processo de cicatrização, apesar de que a ausência de osso não se constitui em uma contra-indicação absoluta para o autotransplante dentário. Osterne, Neto, Lima \& Nogueira (2015) relataram o caso de uma paciente de 14 anos diagnosticada com ameloblastoma em mandíbula, a qual foi tratada com ressecção em bloco e posteriormente reabilitada com autotransplante de terceiros molares imaturos e tratamento ortodôntico, sendo este o primeiro caso de regeneração óssea alveolar em defeitos ósseos mandibulares após ressecção de ameloblastoma e autotransplantações dentárias sem enxerto ósseo. Recentemente, um estudo in vivo demonstrou que células da bainha radicular do epitélio de Hertwig podem estimular a diferenciação osteogênica de células foliculares dentárias por meio comunicação célula a célula e poderia desempenhar um papel na formação óssea alveolar (Yang et al., 2014).

Quando questionados se já haviam assistido ou mesmo realizado a uma cirurgia de autotransplante dentário, apenas 5 graduandos (3,5\%) responderam que sim. Para Parreira (2010), não é comum que esta técnica seja a primeira escolha frente à variedade de tratamentos existentes que visam a substituição de dentes permanentes. Os custos da endodontia (quando necessária após o autotransplante) são comparáveis aos custos de uma reabilitação protética removível unitária e o prognóstico do autotransplante é questionável.

É importante que se faça um questionamento acerca da realidade sobre dentes autotransplantados: por que há um número tão reduzido de casos descritos e apresentados em nosso país? Provavelmente uma das razões seja a falta de profissionais treinados para a realização desta técnica e a falta de conhecimento geral de que se trata de um procedimento com taxa de sucesso favorável, podendo, inclusive, os elementos transplantados serem movimentados ortodonticamente, se necessário (Consolaro, Pinheiro, Intra \& Roldi, 2008). O processo reabilitador com prótese implantossuportada possui uma taxa de sucesso maior que o dente autotransplantado e isso pode ser o principal determinante para a diminuição da realização desse tipo de procedimento. 
Para Correio et al. (2015) o autotransplante dentário é tecnicamente simples, de baixo custo e não necessita de equipamentos especializados. Logo, apresenta-se como uma opção de tratamento reabilitador, principalmente, no Sistema Único de Saúde, onde a maior parcela da população jovem economicamente desfavorecida obtém seu primeiro atendimento.

A literatura aponta como vantagem o fato de esta opção terapêutica requerer usualmente apenas de uma cirurgia. Havendo sucesso do autotransplante, o dente recupera frequentemente a função proprioceptiva, com cicatrização periodontal normal, permitindo uma sensação natural de mastigação e uma resposta biológica natural. Assim, o dente autotransplantado pode ser utilizado como pilar de ponte, em prótese fixa, ou mesmo como ancoragem ortodôntica, mas talvez a vantagem mais relevante, é a sua utilização em jovens em crescimento, uma vez que permite indução continuada do osso alveolar (Santiago, 2012).

Ao serem questionados se existe a necessidade de se realizar ou não o tratamento endodôntico no dente autotransplantado, 64,3\% não souberam responder. Testes de vitalidade pulpar periódicos e ao longo prazo, com o intuito de se comprovar a vitalidade ou a necrose pulpar, bem como exames radiográficos são fatores que determinam a necessidade da endodontia, mesmo o dente estando ainda com ápice aberto (Mikami et al., 2014).

A contenção é um método que há anos vem sendo preconizado nos casos de transplantes dentários. Algumas técnicas para estabilização dos dentes transplantados foram descritas, como fixação com suturas, brackets ortodônticos e fixação não rígida com fio de nylon e resina. Antigamente recomendava-se contenção rígida e por longos períodos. Hoje em dia, sabe-se que a contenção não rígida e por curto período de tempo é mais favorável ao periodonto, permitindo a reinserção das fibras do ligamento periodontal e diminuindo consideravelmente as chances de haver anquilose (Sales, 2014).

Alguns autores defendem que se mantenha a contenção por sete a dez dias, verificando, ao final deste período, se o dente tem mobilidade ou não. Em caso afirmativo, é fixado novamente até ter mobilidade aceitável. A contenção deve incluir os dentes adjacentes ao transplante utilizando resina composta e fios ortodônticos. Durante esse período, recomenda-se ao paciente manter uma dieta suave e uma higiene oral rigorosa, principalmente durante a primeira semana, com o uso de enxaguatórios bucais e géis anti-sépticos (Nieto, 2016). Dos graduandos entrevistados neste trabalho, $71,3 \%$ não soube responder a esta questão. Segundo Hoffelder (2001), esta etapa da cirurgia de transplante é uma das mais contraditórias, já que não há unanimidade entre os vários trabalhos publicados na literatura. Segundo ele, estudos experimentais como o de Sagne (1985), não encontraram nenhuma diferença entre esplintar e não esplintar. 
Para 53,8\% dos entrevistados, a ausência de carga no dente autotransplantado é essencial. Após colocado no alvéolo, o dente deve ser ajustado numa posição de ligeira infra- oclusão, uma vez que este não deve ser submetido a forças oclusais durante o processo de cicatrização. Porém, o dente não deve ser posicionado abaixo do nível ósseo devido ao risco de ocorrer anquilose e de, consequentemente, a erupção natural não acontecer. Quando os dentes autotransplantados apresentarem a raiz completamente desenvolvida, como afirma Oliveira (2012), estes não podem ser deixados numa posição de infra-oclusão devido à sua incapacidade de erupcionar.

\section{Considerações Finais}

Apesar de não ser um procedimento comum na prática clínica e do ensino odontológico, dentro da metodologia empregada nesse estudo, foi possível observar um nível de conhecimento satisfatório dos estudantes de odontologia da UFCG, Patos-Paraíba acerca do autotransplante dentário.

\section{Referências}

Andreasen JO, Schwartz O, Kofoed T \& Daugaard-Jensen J (2009). Transplantation of Premolars as an Approach for Replacing Avulsed Teeth. Pediatric Dentistry, 31(2), 129-32.

Aslan BI, Uçuncu N \& Dogan A (2010). Long-term follow-up of a patient with multiple congenitally missing teeth treated with autotransplantation and orthodontics. Angle Orthod, 80(2), 396-404.

Consolaro A, Pinheiro TN, Intra JBG \& Roldi A (2008). Transplantes dentários autógenos: uma solução para casos ortodônticos e uma casuística brasileira. Revista Dental Press de Ortodontia e Ortopedia Facial, 13(2), 23-8.

Correio DSR, Correio LSC, Correio ELA, Correio HLS, Correio LPSZ \& Correio AFA (2015). Autotransplante dentário: uma opção reabilitadora e viável ao SUS. Revista Bahiana de Odontologia, 1(6), 47-51. 
Donado M (2007). Implantes y traumatismos dentários (3a ed). Masson: ed. Cirurgia Bucal

Duarte ÉEN, Macedo IRR, Carvalho WRS, Oliveira HC, Cristino MR \& Neto NC (2017). Autotransplante dentário: uma alternativa viável para a reabilitação oral. Revista Digital da Academia Paraense de Odontologia, 1(1), 29-34.

Gilijamse M, Baart JÁ, Wolff J, Sándor JK \& Forouzanfar T (2016). Tooth autotransplantation in the anterior maxilla and mandible: retrospective results in young patients. Oral and Maxillofacial Surgery, 122(6), 187-92.

Hoffelder J (2001). Transplantes dentais. Trabalho de Conclusão de Curso, Universidade Federal de Santa Catarina, Florianópolis, Santa Catarina, Brasil.

Kokai S, Kanno Z, Koike S, Uesugi S, Takahashi Y, Ono T \& Soma K (2015). Retrospective study of 100 autotransplanted teeth with complete root formation and subsequent orthodontic treatment. American Journal of Orthodontics \& Dentofacial Orthopedics, 148(6), 982-9.

Mateo Castillo JF, Olano-Dextre TL, Neves LT, Nishiyama CK, Faco RAS \& Pinto LC (2017). Abordagem endodôntica em transplante dentário autógeno em indivíduo com fissura labiopalatina. Revista Cubana de Estomatologia, 54(4), 01-12.

Mikami JR, Filho JRL, Nogueira PTBC, Júnior OB, Júnior EZS \& Filho LLTN (2014). Transplante dental autógeno - relato de caso. Revista de Cirurgia e Traumatologia Bucomaxilo-facial, 4(14), 51-7.

Nagori AS, Bhutia O, Roychoudhury A \& Pandey RM (2014). Immediate autotransplantation of third molars: an experience of 57 cases. Oral Surgery, Oral Medicine, Oral Pathology, Oral Radiology, 118(4), 400-07.

Nieto, JLB (2016). Autotransplante dentario. Trabalho de Conclusão de curso, Universidade de Sevilla, Sevilla, Espanha.

Oliveira, RMA (2012). Autotransplante Dentário. Dissertação, Universidade de Lisboa, Lisboa, Portugual. 
Osterne RLV, Neto JJSM, Lima ADMA \& Nogueira RLM (2015). Autotransplantation of immature third molars and orthodontic treatment after en bloc resection of conventional ameloblastoma. Oral and Maxillofacial Surgery, 73(9), 1686-94.

Parreira, ARCBM (2010). Autotransplante do terceiro molar. Trabalho de Conclusão de curso, Universidade do Porto, Porto, Portugual.

Peixoto, AC; Melo AR \& Santos TS (2013). Transplante dentário: atualização da literatura e relato de caso. Revista de Cirurgia e Traumatologia Buco-maxilo-facial, 13(2), 75-80.

Queiroz NB; Freire HS, Cabral ARA, Alves IFS, Albuquerque AFM \& Esses DFS (2016). Cirurgia de transplante dentário autógeno pela técnica mediata - relato de caso clínico. Jornada Odontológica dos Acadêmicos da Católica - Joac, 2(2), 1-7.

Sales MT (2014). Substituição de incisivo central superior por pré-molar transplantado. Trabalho de conclusão de curso, Faculdade de Ciências da Saúde da Universidade de Brasília, Brasília, Distrito Federal, Brasil;

Santiago, EFA (2012). Avaliação retrospectiva longitudinal de autotransplantes dentários. Dissertação, Faculdade de Medicina Dentária da Universidade do Porto, Porto, Portugual.

Yang, Y; Ge, Y; Chen, G; Yan, Z; Yu, M; Feng, L; Jiang, Z; Guo, W \& Tian, W (2014). Hertwig's epithelial root sheath cells regulate osteogenic differentiation of dental follicle cells through the Wnt pathway. Bone, 63, 158-65.

Yu, HJ; Jia, P; Lv, Z \& Qiu, LX (2017). Autotransplantation of third molars with completely formed roots into surgically created sockets and fresh extraction sockets: a 10-year comparative study. International Journal of Oral and Maxillofacial Surgery, 46(4), 531-38. 
Research, Society and Development, v. 9, n. 7, e641974632, 2020

(CC BY 4.0) | ISSN 2525-3409 | DOI: http://dx.doi.org/10.33448/rsd-v9i7.4632

Porcentagem de contribuição de cada autor no manuscrito

Regina Mendes da Silva - 13\%

Julierme Ferreira Rocha - 12\%

Luanna Abílio Diniz Melquíades de Medeiros - 7\%

Lais Sousa Maia-7\%

Vitor Nascimento Goes $-7 \%$

Ocimar Lopes de Oliveira-7\%

Lukas Natã Mendes Fragoso - 7\%

Ana Beatriz Rodrigues Moura - 7\%

Elizandra Silva da Penha-7\%

Gymenna Maria Tenório Guênes- 7\%

Manuella Santos Carneiro Almeida-7\%

Camila Helena Machado da Costa Figueiredo- $12 \%$ 